\title{
THE ASYMPTOTIC SOLUTION OF AN OPERATIONAL EQUATION*
}

BY

JOHN R. CARSON

The subject of this note is the asymptotic solution of the operational equation

$$
h=\frac{p^{r}}{p-\lambda} .
$$

For the significance of this equation and general methods of solution, the reader is referred to two papers on the Heaviside operational calculus, which appeared in the Bulletin of the American Mathematical Society; one in the January-February 1926 issue by the writer, and the other in the May-June 1927 issue by March. $\dagger$ For present purposes it is sufficient, however, to state that (1) is a purely symbolic equation in which $p$ symbolically represents the differential operator $d / d t$, and that $h=h(t)$, for real values of $t \geqq 0$, is uniquely defined and determined either by the Laplace integral equation (valid for all values of $p$ to the right of $\lambda$ in the complex plane)

$$
\int_{0}^{\infty} h(t) e^{-p t} d t=\frac{1}{p-\lambda} \cdot \frac{1}{p^{1-r}}
$$

or by the Fourier-Bromwich integralf

$$
h(t)=\frac{1}{2 \pi i} \int_{c-i \infty}^{c+i \infty} \frac{e^{t p}}{p-\lambda} \cdot \frac{d p}{p^{1-r}}
$$

In equation (1), $\lambda$ is an unrestricted parameter in the complex plane; without loss of essential generality, however, it will be assumed that $|\lambda|=1$, and $\lambda=e^{i \alpha}$. The exponent $r$ is a proper fraction; thus $0 \leqq r \leqq 1$. For simplicity, $r$ will be assigned the value $\frac{1}{2}$ in the following; the methods of analysis applied to this case are applicable without change to all values of $r$ lying

* Presented to the Society, August 30,1929; received by the editor of the Bulletin in February, 1929, accepted for publication in the Bulletin, and subsequently transferred to the Transactions; revised copy received by the editors of the Transactions in June, 1929.

† A paper by Wiener, The operational calculus, Mathematische Annalen, vol. 95, No. 4, may also be consulted with profit.

$\ddagger$ Bromwich, Proceedings of the London Mathematical Society, 1916, pp. 410-420. Jeffrey, Operational Methods in Mathematical Physics. 
between zero and unity. We shall therefore limit explicit consideration to the equation

$$
h=\frac{p^{1 / 2}}{p-\lambda} .
$$

The operational equation (4) is of considerable importance in the theory of asymptotic solutions of operational equations, because a fairly large class of operational equations is reducible to sums of expressions of the form of (4). In addition it serves to introduce the question as to whether the divergent solution derived by operational processes is a complete asymptotic expansion, or whether an additional term is required.

In accordance with the usual Heaviside process, a divergent solution of (4) is obtained by the following remarkably simple process. Write (4) as

$$
h=-\frac{1}{\lambda}\left(1-\frac{p}{\lambda}\right)^{-1} p^{1 / 2}
$$

and expand by the binomial theorem without reference to convergence; thus

$$
h=-\frac{1}{\lambda}\left(1+\frac{p}{\lambda}+\left(\frac{p}{\lambda}\right)^{2}+\left(\frac{p}{\lambda}\right)^{3}+\cdots\right) p^{1 / 2} .
$$

Now replace $p^{n}\left(n\right.$ integral) by $d^{n} / d t^{n}$ and $p^{1 / 2}$ by $1 /(\pi t)^{1 / 2} ;(5)$ thus becomes

$$
\begin{aligned}
h \sim-\frac{1}{\lambda(\pi t)^{1 / 2}}\left\{1-\frac{1}{2 \lambda t}\right. & +\frac{1 \cdot 3}{(2 \lambda t)^{2}}-\frac{1 \cdot 3 \cdot 5}{(2 \lambda t)^{3}}+\cdots \\
& \left.+(-1)^{n} \frac{1 \cdot 3 \cdot 5 \cdots(2 n-1)}{(2 \lambda t)^{n}} \cdots\right\} .
\end{aligned}
$$

The beautifully simple process sketched above whereby the divergent solution (6) is derived, can be applied to a large class of operational equations in which fractional powers of the operator $p$ appear. Such divergent solutions which are derivable for a large number of operational equations representing physical problems, are true asymptotic solutions. In some cases, however, an extra term must be added, while in most cases the precise asymptotic character of the expansion, and the order of magnitude of the error committed by stopping with the smallest term of the divergent series, require independent investigation. In all cases examined, however, and presumably in all cases, the divergent solution has a real significance, at least for restricted values of the argument $t$. The analysis of equations (1) and (4) serves as an 
excellent illustration of these questions, and is not without interest in its own right.

If we write

(7) $S_{n}=-\frac{1}{\lambda(\pi t)^{1 / 2}}\left\{1-\frac{1}{2 \lambda t}+\frac{1 \cdot 3}{(2 \lambda t)^{2}}+\cdots+(-1)^{n} \frac{1 \cdot 3 \cdot 5 \cdots(2 n-1)}{(2 \lambda t)^{n}}\right\}$,

it is easy to show by aid of either (2) or (3) (setting $r=\frac{1}{2}$ ) that $h$ is equal to $S_{n}$ plus a remainder. The investigation of the remainder directly from (2) or (3) appears to present difficulties. We can, however, easily derive from (2) the explicit solution

$$
h=e^{\lambda t} \int_{0}^{t} \frac{e^{-\lambda r}}{(\pi \tau)^{1 / 2}} d \tau .
$$

That (8) is the solution of (1) and (2) for $r=\frac{1}{2}$ is easily shown as follows. We have the known identities

and

$$
\int_{0}^{\infty} \frac{e^{-p t}}{(\pi t)^{1 / 2}} d t=\frac{1}{p^{1 / 2}}
$$

$$
\int_{0}^{\infty} e^{\lambda t} \cdot e^{-p t} d t=\frac{1}{p-\lambda}
$$

for all values of $p$ to the right of $\lambda$ in the complex plane. From the foregoing equation (8) follows at once from a theorem due to Borel* which may be stated as follows:

If

$$
\begin{aligned}
& \int_{0}^{\infty} f(t) e^{-p t} d t=F_{1}(p) \cdot F_{2}(p), \\
& \int_{0}^{\infty} f_{1}(t) e^{-p t} d t=F_{1}(p), \\
& \int_{0}^{\infty} f_{2}(t) e^{-p t} d t=F_{2}(p),
\end{aligned}
$$

then

$$
f(t)=\int_{0}^{t} f_{1}(t-\tau) f_{2}(\tau) d \tau=\int_{0}^{t} f_{1}(\tau) f_{2}(t-\tau) d \tau .
$$

- Legons sur les Series Divergentes, 1901, p. 104; see also Electric Circuit Theory and the Operational Calculus, New York, McGraw Hill Book Company, p. 41. 
Now suppose that the real part of $\lambda \geqq 0$. For this case we can write

$$
\int_{0}^{t}=\int_{0}^{\infty}-\int_{t}^{\infty}
$$

The first integral on the right hand side is equal to $1 / \lambda^{1 / 2}$, and repeated integration by parts applied to the second integral gives

$$
h=\frac{e^{\lambda t}}{\lambda^{1 / 2}}+S_{n}+R_{n}
$$

where the remainder term is

$$
R_{n}=(-1)^{n} \frac{1 \cdot 3 \cdot 5 \cdots(2 n+1)}{(2 \lambda)^{n+1}} e^{\lambda t} \int_{t}^{\infty} \frac{e^{-\lambda \tau}}{\tau^{n+1}(\pi \tau)^{1 / 2}} d \tau
$$

This remainder term is easily seen to be less than the last term $T_{n}$ of the divergent series; that is, $\left|R_{n}\right|<\left|T_{n}\right|$. Consequently we see that for the case where the real part of $\lambda \geqq 0$, the divergent series (6), directly derived from the operational equation, requires the addition of the extra term $e^{\lambda t} / \lambda^{1 / 2}$, and that $h$ is asymptotically represented by $e^{\lambda t} / \lambda^{1 / 2}+S_{n}$ with an error less than the last term included in the divergent series $S_{n}$. This conclusion, it may be remarked, has long been known.

When the real part of $\lambda$ is negative, the problem is quite different, and the method employed above is not applicable. We can, however, write (8) in the form

$$
h=\frac{1}{\pi^{1 / 2}} \int_{9}^{t} \frac{\dot{e}^{\lambda \tau}}{(t-\tau)^{1 / 2}} d \tau .
$$

For this case it has been shown by Gronwall (in an unpublished memorandum communicated to the writer) that $S_{n}$ is an asymptotic expansion of $h$. Gronwall, however, did not investigate the error committed by terminating the divergent series, nor did he examine the question as to whether the addition of the "extra term" $e^{\lambda t} / \lambda^{1 / 2}$ does or does not contribute to numerical accuracy in computation. It is evident, of course, that when $S_{n}$ is asymptotic to $h, e^{\lambda t} / \lambda^{1 / 2}+S_{n}$ is also asymptotic, and vice-versa.

In a recent paper* it is stated that (9) applies for all values of $\lambda$ (except $\lambda=-1$, in which case the term $e^{\lambda t} / \lambda^{1 / 2}$ is to be omitted), and that the re-

* Asymptotic expansions of Heaviside's operators, Berg, Haefner and Smith, Journal of the Franklin Institute, February, 1928. In one and one case only Heaviside himself (Electromagnetic Theory, vol. II) adds the extra term when the real part of $\lambda$ is negative. His justification of his procedure, however, is exceedingly obscure. 
mainder term is less than the last term of $S_{n}$. This statement is not correct, and the method of analysis employed is invalid. The question, however, admits of simple and rigorous investigation by contour integration, as employed below.

Returning to (8) it may be written as

$$
h=\frac{e^{\lambda t}}{(\pi \lambda)^{1 / 2}} \int_{0}^{t e^{i \alpha}} \frac{e^{-z}}{z^{1 / 2}} d z
$$

representing integration in the complex plane along the straight line $z=e^{i \alpha}$. Since we are concerned with negative values of $\lambda$, we shall, without loss of essential generality, assume that $\lambda$ lies in the second quadrant, and write $\lambda=e^{i \alpha}=-\mu+i \nu$, so that both $\mu$ and $\nu$ are $\geqq 0$.

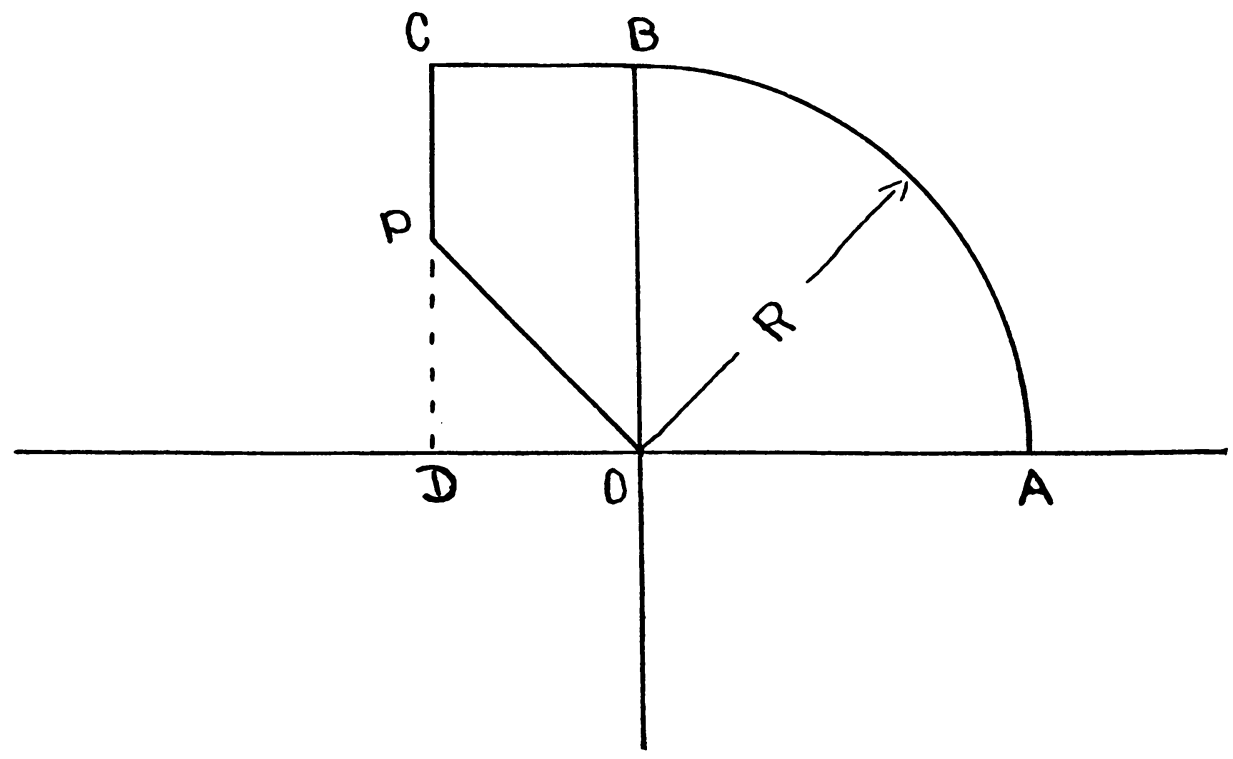

Referring to the accompanying sketch, the integral is along the path $0 P$ where $P=t e^{i \alpha}$. Since the singularity at the branch point $z=0$ contributes nothing, it follows from Cauchy's theroem that

$$
\int_{0}^{P}=\int_{0}^{A}+\int_{A}^{B}+\int_{B}^{C}-\int_{P}^{C}
$$

where $A B$ is the arc of a circle of radius $R$. If $R$ is made infinite it is easy to see that the second and third integrals vanish, and that

$$
\int_{0}^{P-t 0^{i \alpha}}=\int_{0}^{\infty}-\int_{t i i^{i \alpha}}^{i \infty} \text {. }
$$


Along the path $0 A(A \rightarrow \infty)$ set $z=\rho$, where $\rho$ is real. Along the path $P C$, $z=-\mu t+i \rho$ and the limits of integration are $\rho=\nu t$ and $\infty$. Consequently (12) becomes

$$
h=\frac{e^{\lambda t}}{\lambda^{1 / 2}}-i \frac{e^{i \nu t}}{(\pi \lambda)^{1 / 2}} \int_{\nu t}^{\infty} \frac{e^{-i \rho}}{(-\mu t+i \rho)^{1 / 2}} d \rho .
$$

Applying repeated partial integrations to the second integral, we thus get

$$
h=\frac{e^{\lambda t}}{\lambda^{1 / 2}}+S_{n}+R_{n}
$$

where the remainder term $R_{n}$ is given by

$$
R_{n}=i(-1)^{n} \frac{1 \cdot 3 \cdot 5 \cdots(2 n+1)}{2^{n+1}} \cdot \frac{e^{i \nu t}}{(\pi \lambda)^{1 / 2}} \int_{n t}^{\infty} \frac{e^{-i \rho}}{(-\mu t+i \rho)^{n+3 / 2}} d \rho
$$

If we now denote the extra term $e^{\lambda t} / \lambda^{1 / 2}$ by $T_{e} ;$ the last retained term of $S_{n}$ by $T_{n}$; and if we write $n+3 / 2=m$ and $\nu / \mu=\rho$, obvious transformations of (15) lead to the expressions

$$
\begin{aligned}
R_{n} & =-\frac{m-1}{\mu^{m-1}} \cdot \frac{1 \cdot 3 \cdot 5 \cdots(2 n-1)}{(2 t)^{n}(\pi t)^{1 / 2}} e^{\mu t} \cdot I_{n} \cdot T_{e} \\
& =-(m-1)\left(\frac{\lambda}{\mu}\right)^{m-1} e^{i \nu t} I_{n} \cdot T_{n}
\end{aligned}
$$

where

$$
I_{n}=\int_{\rho}^{\infty} \frac{e^{i(m \theta-\mu t x)}}{\left(x^{2}+1\right)^{m / 2}} d x
$$

and

$$
\theta=\cos ^{-1} \frac{1}{\left(x^{2}+1\right)^{1 / 2}} .
$$

The investigation of the remainder term and the asymptotic character of the expansion is thus reduced to a study of the integral $I_{n}$. Equations (16) and (17) conveniently correlate this remainder term with the "extra term" $T_{e}$ and the last retained term $T_{n}$ of the divergent series.

Now when a divergent series is employed for numerical computation it is the usual and logical procedure to stop with that term whose absolute value is the numerically smallest. Instead, therefore, of dealing with $I_{n}$ for unrestricted values of $t$, we shall assign to $t$ the value $n$, so that $T_{n}$, the last 
retained term, is the numerically smallest of the series. This restriction will be understood to apply to the following, and we write

$$
I_{n}=\int_{\rho}^{\infty} \frac{e^{i(m \theta-\mu n x)}}{\left(x^{2}+1\right)^{m / 2}} d x, m=n+3 / 2 .
$$

If $n$ is made sufficiently large, this integral can be evaluated to any required degree of accuracy by writing $x=\rho+y$, and expanding the integrand in the form

$$
\exp \left[-n\left(c_{0}+c_{1} y+c_{2} y^{2}+\cdots\right)\right]
$$

For sufficiently large values of $t=n$, the integral becomes

$$
\frac{1}{2 n} e^{\left(\beta-n c_{0}\right)}\left\{\left(\frac{\pi n}{c_{0}}\right)^{1 / 2}-\frac{n c_{1}}{c_{2}}\left(1-\frac{\beta}{3 \cdot 1 !}+\frac{\beta^{2}}{5 \cdot 2 !} \cdots\right)\right\}
$$

where $\beta=n c_{1}^{2} /\left(4 c_{2}\right)$. In this way we find, without going through straightforward details, that for $1>\rho>0$,

$$
\left|R_{n}\right| \rightarrow \frac{T_{n}}{\left\{2\left[1-\left(1+\rho^{2}\right)^{-1 / 2}\right]\right\}^{1 / 2}}
$$

with increasing values of $n$. On the other hand, for any fixed large value of $n$, it is always possible to choose a value of $\rho$ so small that

$$
\left|R_{n}\right|=\left|T_{n}\right|(\pi n / 2)^{1 / 2} \text {. }
$$

In the foregoing we have supposed that the index $n=t$ is a large number. In practical applications, however, we are interested in relatively small values of $n$. For such cases the only way of evaluating the integral $I_{n}$ is by the pedestrian process of numerical or mechanical quadrature. Fortunately however, the convergence of the integrand is so rapid as to make this process relatively easy and accurate. For the case $n=5, T_{n}=T_{5}$ is approximately $1 \%$ of the leading term. The results of numerical integration for this case are tabulated below.

$\begin{array}{ccc}\rho & \left|R_{n}\right| /\left|T_{n}\right| & \left|R_{n}+T_{e}\right| /\left|R_{n}\right| \\ 0 & 2.95 & 0.30 \\ 0.1 & 2.50 & 0.40 \\ 0.2 & 2.14 & 0.68 \\ 0.3 & 1.86 & 1.08 \\ 0.4 & 1.65 & 1.65 \\ 0.5 & 1.50 & 2.45 \\ 0.8 & 1.20 & 6.60 \\ 1.0 & 1.10 & 10.60\end{array}$


From the foregoing we conclude as follows:

(1) In the region $0 \leqq \rho \leqq 1,\left|R_{n}\right|$ is everywhere greater than $\left|T_{n}\right|$. The ratio $\left|R_{n}\right| /\left|T_{n}\right|$ increases with decreasing values of $\rho$ and with increasing values of $n$. In this region, therefore, the error committed by stopping with the term $T_{n}$ is greater than that term.

(2) If $\rho$ is less than a certain value $\rho(n)$, depending on the values of $t=n$ and decreasing therewith, $\left|T_{c}+R_{n}\right|<\left|R_{n}\right|$. In this region, therefore, the omission of the extra term $T_{0}$ contributes to numerical accuracy. For $n=5$, $\rho(n)=0.3$ approximately, and as $n$ is made indefinitely large, $\rho(n)$ approaches zero. Note, however, that as $n \rightarrow \infty$, the value of the extra term becomes negligible compared with $S_{n}$.

The foregoing will now be applied to deriving Heaviside's "generalized exponential series."

If the operational equation (4) is expanded in the series

$$
h=\frac{1}{p^{1 / 2}}+\frac{\lambda}{p p^{1 / 2}}+\frac{\lambda^{2}}{p^{2} p^{1 / 2}}+\cdots,
$$

the solution, in accordance with the well known methods of the operational calculus, is then

$$
h=2\left(\frac{t}{\pi}\right)^{1 / 2}\left\{1+\frac{1}{3}(2 \lambda t)+\frac{1}{3 \cdot 5}(2 \lambda t)^{2}+\cdots\right\} .
$$

But it has been shown that

$$
\begin{aligned}
h & \sim \frac{e^{\lambda t}}{\lambda^{1 / 2}}+S_{n} \\
& \sim \frac{e^{\lambda t}}{\lambda^{1 / 2}}-\frac{1}{\lambda(\pi t)^{1 / 2}}\left(1-\frac{1}{2 \lambda t}+\frac{1 \cdot 3}{(2 \lambda t)^{2}}-\cdots\right)
\end{aligned}
$$

for all complex values.

Equating we get

$$
e^{\lambda t} \sim \sum_{m=-n}^{\infty} \frac{(\lambda t)^{m-1 / 2}}{\Gamma(m+1 / 2)},
$$

the expansion for negative values of $m$ being divergent but asymptotic.

More generally, from the operational equation

$$
\begin{array}{rlrl}
h & =\frac{p^{r}}{p-\lambda} & \left(0<l r_{0}<\mathbf{1} 1\right) \\
e^{\lambda t} & \sim \sum_{m=-n}^{\infty} \frac{(\lambda t)^{m-r}}{\Gamma(m+1-r)} .
\end{array}
$$


In accordance with the foregoing analysis the expansion ceases to have any significance as $\lambda$ approaches -1 .

This paper will now be closed by a very brief discussion of what $I$ have termed the Heaviside Rule; it is as follows:

If the operational equation $h=1 / H(p)$ admits of formal series expansion

$$
\begin{aligned}
h= & a_{0}+a_{1} p^{1 / 2}+a_{2} p+a_{3} p p^{1 / 2}+\cdots \\
& +a_{2 n-1} p^{n} p^{1 / 2}+a_{2 n} p^{n}+\cdots,
\end{aligned}
$$

then the equation has a solution, usually divergent and asymptotic,

$$
h(t) \sim a_{0}+\left(a_{1}+a_{3} \frac{d}{d t}+a_{5} \frac{d^{2}}{d t^{2}}+\cdots+a_{2 n-1} \frac{d^{n}}{d t^{n}}+\cdots\right) \frac{1}{(\pi t)^{1 / 2}} .
$$

To establish this result and the restrictions imposed on its generality we proceed as follows:

The integral equation of the problem

$$
\int_{0}^{\infty} h(t) e^{-p t} d t=\frac{1}{p H(p)}
$$

cannot be employed directly; instead we start with the auxiliary equation defining the auxiliary function $g(t)$ :

$$
\int_{0}^{\infty} g(t) e^{-p t} d t=\frac{1}{p^{n+1} H(p)}
$$

It will be evident at once that $h(t)=\left(d^{n} / d t^{n}\right) g(t)$. Now we can always write, irrespective of the region of convergence of the expansion,

$$
\begin{aligned}
\int_{0}^{\infty} g(t) e^{-p t} d t=\frac{1}{p^{n+1}}\left(a_{0}+a_{1} p^{1 / 2}+a_{2} p\right. & +\cdots \\
& \left.+a_{2 n-1} p^{n} p^{1 / 2}+a_{2 n} p^{n}\right)+\frac{1}{p^{n+1}} R_{n}(p),
\end{aligned}
$$

where $R_{n}(p)$ is the remainder in the series expansion of $1 / H(p)$. Remembering that $h=\left(d^{n} / d t^{n}\right) g$ we have, by well established processes,

$$
h(t)=a_{0}+\left(a_{1}+a_{3} \frac{d}{d t}+a_{5} \frac{d^{2}}{d t^{2}}+\cdots+a_{2 n+1} \frac{d^{n}}{d t^{n}}\right) \frac{1}{(\pi t)^{1 / 2}}+r_{n}(t),
$$

where $r_{n}(t)=\left(d^{n} / d t^{n}\right) \rho_{n}(t)$ and $\rho_{n}$ is defined by the equation

$$
\int_{0}^{\infty} \rho_{n}(t) e^{-p t} d t=\frac{1}{p^{n+1}} R_{n}(p) .
$$


We observe that by hypothesis $\left(1 / p^{n+1}\right) R_{n} \sim a_{2 n+1} / p^{1 / 2}$ as $p \rightarrow 0$; if we can show correspondingly that $\rho_{n}(t) \sim a_{2 n+1} /(\pi t)^{1 / 2}$ as $t \rightarrow \infty$, then $r_{n}=O\left(t^{-(2 n+3) / 2}\right)$ and the asymptotic character of the expansion is established. The required restrictions on the function $H(p)$ are given by the following proposition:

If in the integral equation

$$
\int_{0}^{\infty} f(t) e^{-p t} d t=F(p)
$$

the function $F(p)$ is everywhere finite and continuous in the right hand side of the complex plane, including the axis of imaginaries, except at $p=0$, in the neighborhood of which $F(p) \sim p^{1 / 2}$, then $f(t) \sim 1 /(\pi t)^{1 / 2}$ as $t \rightarrow \infty$.

In the light of the foregoing, the Heaviside Rule leads to a valid asymptotic solution of the operational equation provided $1 / H(p)$ has no singularities on the axis of imaginaries or to the right thereof. Fortunately in the application of the operational calculus to physical problems this condition is satisfied in a great majority of cases, and in the rare cases where it is not, this is usually immediately evident from the physical problem itself. Consequently the engineer and physicist can rely on the Heaviside Rule with confidence.

It will be noted, however, that the foregoing discussion merely establishes the asymptotic character in the sense of Poincaré, and does not determine the magnitude of the error committed by stopping with the smallest term (for finite values of $t$ ), nor in particular the effect on numerical accuracy of including "extra" terms corresponding to singularities in $1 / H(p)$ to the left of the imaginary axis. Such questions depend, for their answer, upon an independent and usually difficult investigation in each specific case. Furthermore it is a reasonable inference from the analysis in the earlier part of this paper that the inclusion of such extra terms may or may not contribute to numerical accuracy depending on the location of the corresponding singularity in the complex plane. To attempt to include such terms in each case would mean giving up the beautiful simplicity and directness of the Heaviside process for a doubtful gain in numerical accuracy and a very small gain at best in the region where the expansion can be employed with numerical precision.

March (Bulletin of the American Mathematical Society, May-June, 1927) briefly discusses the general problem dealt with above by aid of the FourierBromwich integral (3), and derives the divergent expansion but does not attempt to discuss the magnitude of the error nor to establish the formal asymptotic character of the expansion. Wiener (The operational calculus, 
Mathematische Annalen, 1926, and appendix to Operational Circuit Analysis by $\mathrm{V}$. Bush) discusses the problem in detail and sets up criteria for a true asymptotic expansion. As Wiener himself observes, however, the operational calculus he deals with differs under certain circumstances from that of Heaviside. For a third and quite different treatment of the problem, the reader is referred to a paper by Tibor v. Stacho (Operatoren Kalkul und Laplacesche Transformation, publication of the Hungarian University of Francis Joseph, VI, 15, 1927).

American Telephone and Telegraph Company, NEW York, N. Y. 